\title{
Cardiotoxic Overdose Treated with Intravenous Fat Emulsion and High-Dose Insulin in the Setting of Hypertrophic Cardiomyopathy
}

\author{
Samuel J. Stellpflug • Sandy J. Fritzlar • Jon B Cole • \\ Kristin M. Engebretsen • Joel S. Holger
}

Published online: 21 December 2010

(C) American College of Medical Toxicology 2010

\begin{abstract}
High-dose insulin (HDI) and intravenous fat emulsion (IFE) are used in overdoses, although rarely combined. To our knowledge, IFE therapy has not been reported in overdoses of diltiazem, metoprolol and amiodarone. We report a severe overdose of these drugs treated with HDI and IFE in a patient with hypertrophic cardiomyopathy (HCM). We also discuss the potential clinical implications of the inotropic effects of HDI in the setting of HCM and the use and efficacy of IFE in this overdose.
\end{abstract}

Keywords Beta blockers · Calcium channel blockers . Intralipid $\cdot$ IFE $\cdot$ HDI $\cdot$ Hypertrophic cardiomyopathy

\section{Introduction}

Intravenous fat emulsion (IFE) and high-dose insulin (HDI) have been increasingly reported in treatment of overdoses,

\footnotetext{
S. J. Stellpflug $(\bowtie) \cdot J$. B. Cole $\cdot$ K. M. Engebretsen · J. S. Holger Clinical Toxicology Service and Department of Emergency Medicine, Regions Hospital, 640 Jackson Street, St. Paul, MN 55101, USA

e-mail: samuel.j.stellpflug@healthpartners.com

J. B. Cole

e-mail: jonbcole@gmail.com

K. M. Engebretsen

e-mail: Kristin.m.engebretsen@healthpartners.com

J. S. Holger

e-mail: joel.s.holger@healthpartners.com

S. J. Fritzlar

Department of Emergency Medicine, United Hospital,

St. Paul, MN, USA

e-mail: sfj0001@gmail.com
}

but they are rarely used in combination with each other [14]. Metoprolol, a $\beta_{1}$-specific beta-adrenergic blocker, is a potentially lethal cardiac toxin in overdose [5]. Diltiazem, a calcium channel-blocking agent that has effects both on the heart and peripheral vasculature, has also proven itself lethal $[6,7]$. Amiodarone exerts its effects through a complex mechanism that includes potassium and sodium channel blockade along with beta-blocker and calcium channel blocker-like effects at the SA and AV node [8]. All three of these drugs can cause life-threatening hypotension primarily through cardiodepressant effects. We report a lifethreatening overdose of diltiazem, metoprolol, and amiodarone successfully treated with IFE and HDI with serum drug levels included. This represents the initial report of IFE use for these specific drugs and includes a discussion of the potential of HDI-induced inotropy causing obstructive cardiac pathophysiology in the unique setting of hypertrophic cardiomyopathy (HCM).

\section{Case Report}

A 30-year-old woman presented to the emergency department (ED) for right lower quadrant abdominal pain. Her past medical history included hypertrophic cardiomyopathy (HCM) with a previously placed automatic implantable cardioverter defibrillator. Initial vital signs included a blood pressure (BP) of $89 / 46 \mathrm{mmHg}$ and a heart rate (HR) of $73 \mathrm{bpm}$. ECG showed a paced rhythm with no change in interval/segment length as compared to a previous ECG. Over the subsequent $3 \mathrm{~h}$, she was evaluated thoroughly for the abdominal pain which included serum and urine laboratory tests, repeated physical examinations, ultrasound, and a computed tomography scan. All of the tests were negative for acute pathology. They did not explain the 
abdominal pain nor the initial borderline hypotension and worsening vital signs that developed over the course of the evaluation. The basic metabolic panel revealed an initial glucose of $123 \mathrm{mg} / \mathrm{dL}$ and a bicarbonate of $20 \mathrm{mEq} / \mathrm{L}$. The patient became confused and the BP and HR dropped to 64/ $41 \mathrm{mmHg}$ and $70 \mathrm{bpm}$ over this initial $3 \mathrm{~h}$ in the ED despite 2 L of intravenous (IV) normal saline (NS). A Foley catheter was placed and no production of urine was evident over the time frame of her clinical decline. At this time in the course of the evaluation, she admitted to taking all of her diltiazem, metoprolol, and amiodarone about $6 \mathrm{~h}$ prior to arrival, and that she never had abdominal pain. Another $2 \mathrm{~L}$ of NS, 6 amp of calcium gluconate $(27 \mathrm{mEq})$, and an HDI bolus of $0.5 \mathrm{U} / \mathrm{kg}$ with an infusion escalated over an hour to $10 \mathrm{U} / \mathrm{kg} / \mathrm{h}$ were administered. A medical toxicologist was consulted at this time. The patient remained hypotensive, confused, and anuric. The central venous pressure was $20 \mathrm{mmHg}$ and an echocardiogram (echo) showed worsening cardiac function. The fluid, calcium, and HDI therapy lasted approximately $85 \mathrm{~min}$ before administering IFE. The glucose provided to the patient over that time period totaled $25 \mathrm{~g}$, coming in the form of $1 \mathrm{amp}$ of D50 at the outset of HDI treatment (fingerstick glucoses remained normal throughout). IFE $(20 \%)$ was given as a $100-\mathrm{mL}$ bolus and an infusion of $1.5 \mathrm{~L}$ over $1 \mathrm{~h}$. Within 15 min of the completion of the bolus, the BP was 110/ $60 \mathrm{mmHg}$, her confusion improved, and the EF returned to her baseline. The patient's baseline echo from prior to this ED visit revealed hypertrophic cardiomyopathy with an ejection fraction (EF) of $55 \%$. A formal cardiac echo was done in the ED starting when the HDI therapy was initiated. The technician stayed in the ED until after the IFE bolus had been infused and the patient had clinically improved. There were no recorded images during this period until the latter portion of the study, so no formal reading was obtained other than post-IFE. Qualitative rather than quantitative data was obtained by the technician and two ultrasound-trained emergency physicians who were present for the exam. The initial images when the patient was in extremis and in the first 10-15 min of the HDI treatment showed the cardiac walls to be hypertrophic and globally hypokinetic. The images just prior to initiation of the IFE showed the EF to be decreased significantly compared to the previous images. A formally read echo, recorded immediately post-IFE administration and during clinical improvement, showed slight hypertrophy and an EF back to baseline $(55 \%)$. The patient was admitted to the ICU, had a stable hospital course, and was transferred to the care of psychiatry on hospital day 5. The prolonged hospital course included fluid and other supportive cares for her renal insufficiency, social services, and psychiatry consultations. Serum levels from the initial ED blood draw were diltiazem, $1,449 \mathrm{ng} / \mathrm{mL}$ (therapeutic 130
190); metoprolol, $388 \mathrm{ng} / \mathrm{mL}$ (30-300); and amiodarone, $2.7 \mathrm{mg} / \mathrm{L}(0.5-2)$.

\section{Discussion}

As previously mentioned, reports of combined IFE/HDI use are rare, as are reports of IFE use in non-comatose patients $[1,2,4]$. Furthermore, IFE use with the specific overdoses reported in this case has not been reported to our knowledge. HDI, along with adequate supportive care including IV NS and calcium, is considered to be among primary therapies for overdoses of both beta-blockers and calcium channel blockers. HDI may have been ineffective in this case due to the presence of HCM. Cardiogenic shock, in the setting of HCM, is typically treated with a combination of IV fluid to maximize ventricular distention and peripheral alpha agonists to increase SVR. This is important especially in HCM with obstructive pathology (HCOM) as the fluids and vasoconstriction decrease the left ventricular outflow obstruction [9]. Insulin exerts its effects through inotropy rather than peripheral vasopressor activity. In fact, it is a potent inotropic agent and vasodilator [10, 11]. Inotropic therapy has been shown to increase the outflow obstruction in HCOM and even induce it in cases of HCM where there is no baseline obstruction, as in our patient (she did not demonstrate obstruction on a previous echo). This could explain why the administration of HDI in this patient did not result in clinical improvement within the 85 min of treatment prior to IFE administration.

IFE, on the other hand, was associated with rapid improvement. Echocardiography was performed simultaneously with the IFE bolus and infusion. The initial images showed low ejection fraction and a small amount of mitral valve insufficiency, and by the end of the infusion the ejection fraction had returned to the patient's baseline compared to a previous echo. The $\log P$ octanol/water partition coefficient $(\log P)$ of a drug may correlate in part with the effectiveness of IFE (higher $\log P$ possibly indicating higher effectiveness). The $\log P$ for diltiazem, metoprolol, and amiodarone are 2.7, 1.88, and 7.8, respectively [12]. These are similar to $\log P$ values for drug overdoses reported to be effectively treated with IFE $[1,2]$. The serum drug levels suggest that a significant portion of the clinical severity was probably due to the diltiazem. There is a case report with a higher serum level of diltiazem treated successfully with other methods, but the patient in this report did not have a cardiomyopathy and the combination of the metoprolol and amiodarone [13]. The likely predominance of the diltiazem effects in this overdose theoretically makes the observed treatment result reasonable due to the $\log P$ of 2.7 , as compared with the 1.88 of metoprolol, and is closer to the values of other 
apparent IFE-sensitive drugs (nebivolol 3.2, verapamil 3.8 , etc.) $[2,4]$.

\section{Conclusion}

To our knowledge, this is the initial report of the use of IFE for any diltiazem, metoprolol, or amiodarone overdose, and represents an addition to the rare reports of the combination of IFE/HDI therapy. The effect of the HDI therapy was likely less than optimal due to the inotropy and vasodilation induced by HDI being counterproductive in the setting of hypertrophic cardiomyopathy. The treatment combination, however, was successful for this patient in reversing severe drug-induced cardiogenic shock.

\section{References}

1. Cave G, Harvey M (2009) Intravenous lipid emulsion as antidote beyond local anesthetic toxicity: a systematic review. Acad Emerg Med 16:815-824

2. Jamaty C, Bailey B, Larocque A, Notebaert E, Sanogo K, Chauny J (2010) Lipid emulsions in the treatment of acute poisoning: a systematic review of human and animal studies. Clin Toxicol $48: 1-27$
3. Shepherd G (2006) Treatment of poisoning caused by betaadrenergic and calcium-channel blockers. Am J Health Syst Pharm 63:1828-1835

4. Stellpflug S, Harris C, Engebretsen K, Cole J, Holger J (2010) Intentional overdose with cardiac arrest treated with intravenous fat emulsion. Clin Toxicol 48(3):227-229

5. Rohrig T, Rundle D, Leifer W (1987) Fatality resulting from metoprolol overdose. J Anal Toxicol 11:231-232

6. Cantrell F, Williams S (2005) Fatal unintentional overdose of diltiazem with antemortem and postmortem values. Clin Toxicol 43:587-588

7. Schoffstall J, Spivey W, Gambone L, Shaw R, Sit S (1991) Effects of calcium channel blocker overdose-induced toxicity in the conscious dog. Ann Emerg Med 20:1104-1108

8. Goddard C, Whorwell P (1989) Amiodarone overdose and its management. Br J Clin Pract 43:184-186

9. Maron B (2002) Hypertrophic cardiomyopathy: a systematic review. JAMA 287:1308-1320

10. Von Lewinski D, Bruns S, Walther S, Kogler H, Pieske B (2005) Insulin causes $\left[\mathrm{Ca}^{2+}\right]_{\mathrm{i}}$-dependent and $\left[\mathrm{Ca}^{2+}\right]_{\mathrm{i}}$-independent positive inotropic effects in failing human myocardium. Circulation 111:2588-2595

11. Holger JS, Engebretsen KM, Fritzlar SJ, Patten LC, Harris CR, Flottemesch TJ (2007) Insulin versus vasopressin and epinephrine to treat beta-blocker toxicity. Clin Toxicol 45:96-401

12. Moffat A, Osselton M, Widdop B, Galichet L (eds) (2004) Part 2, monographs. In: Clarke's analysis of drugs and poisons in pharmaceuticals, body fluids, and postmortem material, 3rd edn. Pharmaceutical Press, London, pp 567-1721

13. Holstege CP, Kirk MA, Furbee RB, Wermuth ME (1998) Wide complex dysrhythmia in calcium channel blocker overdose responsive to sodium bicarbonate therapy. J Toxicol Clin Toxicol 36:509 